\title{
Factors predicting the response to oral fluoropyrimidine drugs: A phase II trial on the individualization of postoperative adjuvant chemotherapy using oral fluorinated pyrimidines in stage III colorectal cancer treated by curative resection (ACT-01 Study)
}

\author{
TAKEO MORI ${ }^{1}$, MASAYUKI OHUE ${ }^{2}$, YASUMASA TAKII ${ }^{3}$, TADASHI HASHIZUME ${ }^{4}$,

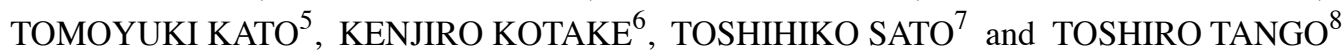 \\ ${ }^{1}$ Tokyo Metropolitan Cancer and Infectious Disease Center, Komagome Hospital, Tokyo; \\ ${ }^{2}$ Osaka Medical Center for Cancer and Cardiovascular Diseases, Osaka; ${ }^{3}$ Niigata Cancer Center Hospital, \\ Niigata; ${ }^{4}$ Mutsu General Hospital, Aomori; ${ }^{5}$ Aichi Cancer Center Hospital, Nagoya; ${ }^{6}$ Tochigi Cancer Center, \\ Utsunomiya; ${ }^{7}$ Yamagata Prefectural Central Hospital, Yamagata; ${ }^{8}$ Center for Medical Statistics, Tokyo, Japan
}

Received August 8, 2012; Accepted October 18, 2012

DOI: 10.3892/or.2012.2177

\begin{abstract}
We evaluated the predictive relevance of several biomarkers on the survival of patients with stage III colorectal cancer treated with adjuvant chemotherapy of oral fluoropyrimidines. This was a multicenter phase II trial on adult patients with histologically confirmed resected stage III (Dukes' C) colorectal cancer. Patients received oral doxifluridine $\left(800 \mathrm{mg} / \mathrm{m}^{2} /\right.$ day) in 3 divided doses, or oral uracil/ tegafur (UFT) $\left(400 \mathrm{mg} / \mathrm{m}^{2} /\right.$ day) in 2 divided doses for 5 days, every 7 days for 12 months with a 5-year follow-up. Outcome measures were disease-free survival and tissue markers [thymidine phosphorylase (TP), dihydropyrimidine dehydrogenase (DPD) protein levels and TP, DPD, thymidylate synthase (TS) and orotate phosphoribosyltransferase (OPRT) mRNA levels in tumor samples and TS tandem-repeat type in blood samples]. There was a significant association between the intratumoral TP/DPD enzyme ratio and disease-free survival when the model included the drug, the parameter and the interactions between them [hazard ratio $(\mathrm{HR})=2.76$; $\mathrm{P}=0.00469]$. The 5-year disease-free survival rate was statistically significantly higher in patients with high TP/DPD ratios [median $\geq 2.63$ : 71.9\%; 95\% confidence interval (CI) 61.480.0] compared to patients with low TP/DPD ratios $(<2.63$ : 57.0\%; 95\% CI 46.3-66.3) (log-rank $\mathrm{P}=0.0277$ ) following
\end{abstract}

Correspondence to: Dr Takeo Mori, Tokyo Metropolitan Cancer and Infectious Disease Center, Komagome Hospital, 3-18-22 Honkomagome, Bunkyo-ku, Tokyo 113-8677, Japan

E-mail: m.takeo@cick.jp

Key words: biomarkers, predictive markers, thymidine phosphorylase/dihydropyrimidine dehydrogenase ratio, disease-free survival adjuvant therapy with oral fluoropyrimidines. No significant association was observed between the intratumoral TP/DPD enzyme ratio (cut-off value 2.0) and the disease-free survival rate in the doxifluridine group; primary endpoint (log-rank $\mathrm{P}=0.6850$ ). The magnitude of the intratumoral TP/DPD enzyme ratio may be a potential indicator for the individualization of postoperative adjuvant chemotherapy with oral fluoropyrimidines for stage III colorectal cancer.

\section{Introduction}

The age-adjusted incidence of colon cancer in Japan in 2003 was second compared to stomach cancer in men $(63.8 / 100,000$ individuals) and breast cancer in women (35.9/100,000 individuals) (1). However, it is estimated that there will be 512,225 cases of colon cancer in Japan in 2020, surpassing the number of breast cancer cases from 2010 onwards as the most common type of cancer in women (1).

Adjuvant chemotherapy improves the overall survival in patients with resected stage III colon cancer (2). In the late 1990 's, intravenous 5-fluorouracil/leucovorin (5-FU/LV) was established as the standard adjuvant treatment for patients with stage III colon cancer (3-5). Since then several oral fluoropyrimidines [i.e. capecitabine, uracil/tegafur (UFT) plus LV] have been demonstrated as an effective alternative to $5-\mathrm{FU} / \mathrm{LV}$ in the treatment of colon cancer $(6,7)$.

Intratumoral expression of the metabolizing enzymes thymidine phosphorylase (TP), dihydropyrimidine dehydrogenase (DPD), thymidylate synthase (TS) and orotate phosphoribosyltransferase (OPRT) are important for the clinical activity of the drugs and may be predictive markers used to guide decision-making regarding the treatment for individual patients. For example, the TP/DPD ratio is significantly higher in cancer cell lines with high sensitivity to doxifluridine (an intermediate metabolite of capecitabine) 
compared to those with lower sensitivity and the possibility of predicting the effectiveness of doxifluridine from the TP/DPD ratio has been demonstrated $(8,9)$. The OPRT/DPD ratio may be a promising predictive marker for UFT (data reported in UFT plus LV) (10).

We conducted a multicenter phase II study on 204 stage III colorectal cancer patients to identify potential biomarkers predictive of outcome to adjuvant therapy with oral fluoropyrimidines. Patients were treated orally for 12 months (11) with doxifluridine or UFT, which were the standard drug treatments in Japan in 2001 when the study was initiated.

\section{Patients and methods}

Patients. Patients with histologically diagnosed stage III (i.e. Dukes' C) colorectal cancer who had undergone curative resection within the last 6 weeks prior to the start of the study were enrolled. Other inclusion criteria were: age 20 to 75 years, Eastern Cooperative Oncology Group (ECOG) performance status of $0-2$, able to take oral medication, not treated with any prior therapy other than surgical resection and adequate organ function. Exclusion criteria were: cancer of the appendix or anal canal derived from the anal glands, synchronous or metachronous cancers or multiple invasive colon cancers (excluding intramucosal cancer).

Patients were recruited from 13 institutions belonging to the Japanese Society for Cancer of the Colon and Rectum which achieved a consensus on the appropriate surgical procedure and follow-up.

The study protocol was approved by the institutional review boards of each participating institution. The study was in accordance with the Ethical Guidelines for Clinical Studies of the Health, Labor and Welfare Ministry in Japan and was conducted in compliance with the Declaration of Helsinki. All patients provided written informed consent.

Study design. Patients were randomly assigned (1:1 ratio) to treatment groups. Tumor site (colon vs. rectosigmoid-upper rectum vs. lower rectum), depth of invasion $[(\mathrm{sm} / \mathrm{mp} / \mathrm{ss}, \mathrm{al}) \mathrm{vs}$. (se, a2/si, ai)], lymph node metastasis (n1 vs. n2/n3) and the study site were selected as stratification factors.

Treatment. Patients received oral doxifluridine $\left(800 \mathrm{mg} / \mathrm{m}^{2} /\right.$ day $)$ in 3 divided doses or oral UFT ( $400 \mathrm{mg} / \mathrm{m}^{2} /$ day) in 2 divided doses for 5 days with 2 rest days and repeated weekly for 12 months. Patients were then followed up until confirmation of recurrence. On the occurrence of adverse events, dose reductions or temporary treatment interruptions were performed as per protocol.

Tissue samples and analysis. Sixty milligrams $\left(5 \mathrm{~mm}^{2}\right)$ of tissue was obtained from each resected tumor, from the marginal portion of the primary lesion and not including necrotic tissue. The sample tissue was divided in half, frozen in liquid nitrogen and stored at $-80^{\circ} \mathrm{C}$ or colder. For analysis of TS tandem repeat type, a $1 \mathrm{ml}$ blood sample was collected within 3 months after surgery and stored at $-80^{\circ} \mathrm{C}$ or colder.

Tissue specimens were sent to Nippon Roche Co., Ltd. (now Chugai Pharmaceutical Co., Ltd.) Research Center for analysis. TP and DPD protein levels were measured by ELISA $(12,13)$ and TP, DPD, TS and OPRT mRNA levels were measured by RT-PCR using a LightCycler ${ }^{\circledR}$ (Roche Diagnostics KK, Tokyo, Japan). Identification of TS tandem repeat type was conducted by PCR-RFLP assay (14).

Evaluation. Physical examination, ultrasonography, chest $\mathrm{X}$-rays and tumor marker measurements were performed before the beginning of the study treatment; every 4 months for the first year after surgery and every 6 months from the second year onwards. Suspected recurrence was confirmed via barium enema, CT or other appropriate diagnostic imaging modality.

Safety was evaluated from reports of adverse events, laboratory examination results and measurements of vital signs. Adverse events were classified according to the Common Toxicity Criteria of the National Cancer Institute (NCI-CTC, version 2). All patients were followed up for a maximum of 5 years until death, failure to follow up or completion of the study.

Study objectives. The primary objective was to examine the effect of the intratumoral TP/DPD enzyme ratio (cut-off value 2.0) on disease-free survival in the doxifluridine group. There were 3 secondary endpoints, which were examined on an exploratory basis: i) Effects of TP and DPD protein levels in tumor samples and the magnitude of the TP/DPD ratio on disease-free and overall survival with oral fluoropyrimidine adjuvant therapy. ii) Effects of TP, DPD, TS and OPRT mRNA levels in tumor samples and the magnitude of the TP/DPD ratio on disease-free and overall survival with oral fluoropyrimidine treatment. iii) Effects of the TS tandem repeat type from blood samples on disease-free and overall survival with oral fluoropyrimidine treatment.

Statistical analysis. Data were analyzed for the full analysis set, comprising all patients who started protocol treatment and in which TP and DPD protein levels were measured.

Time-to-event endpoints were analyzed using the KaplanMeier method and 5-year survival rates were estimated with 95\% confidence intervals (CI). Differences between patient cohorts were examined with the log-rank test. Disease-free survival was defined as the period from the date of enrollment to the date of confirmation of either recurrence or death, whichever preceded. Recurrence was defined as the occurrence of metachronous colon cancer or secondary invasive cancer. Patients without recurrence or death at the time of analysis were censored at the final observation time. Overall survival was defined as the period from the date of enrollment to the date of confirmation of death from any cause. In the analysis of overall survival, patients who survived were censored at the final observation time.

Proportional hazard models were used for the analysis of disease-free and overall survival. We examined 3 different models: drug and the parameter (e.g., TP/DPD ratio), drug and the parameter and the interactions between them and stratification factors as covariates.

We estimated hazard ratios (HRs) and a two-sided Wald P-value for each model. A P-value of $\leq 0.05$ was used to determine whether or not a factor had predictive value.

Further post-hoc exploratory analyses were performed. A Cox regression analysis was performed to identify a cut-off value for the intrartumoral TP/DPD enzyme ratio to distin- 
Table I. Patient baseline characteristics.

\begin{tabular}{|c|c|c|}
\hline & $\begin{array}{l}\text { Doxifluridine } \\
\quad(n=102)\end{array}$ & $\begin{array}{l}\text { Uracil/tegafur } \\
\quad(n=102)\end{array}$ \\
\hline & $\mathrm{n}(\%)$ & $\mathrm{n}(\%)$ \\
\hline \multicolumn{3}{|l|}{ Age, years } \\
\hline Median (range) & $63(26-75)$ & $62.5(33-75)$ \\
\hline \multicolumn{3}{|l|}{ Gender } \\
\hline Male & $55(53.9)$ & $60(58.8)$ \\
\hline Female & $47(46.1)$ & $42(41.2)$ \\
\hline \multicolumn{3}{|l|}{ Tumor location } \\
\hline Colon & $59(57.8)$ & $57(55.9)$ \\
\hline Rectum & $43(42.2)$ & $45(44.1)$ \\
\hline \multicolumn{3}{|l|}{ Histological type } \\
\hline Well differentiated & $35(34.3)$ & $32(31.4)$ \\
\hline Moderately differentiated & $62(60.8)$ & $66(64.7)$ \\
\hline Poorly differentiated & $4(3.9)$ & $3(2.9)$ \\
\hline Mucinous & $1(1.0)$ & $1(1.0)$ \\
\hline \multicolumn{3}{|l|}{ Depth of invasion ${ }^{a}$} \\
\hline $\mathrm{sm}, \mathrm{mp}$ & $13(12.7)$ & $12(11.8)$ \\
\hline ss, a1 & $53(52.0)$ & $57(55.9)$ \\
\hline se, a2 & $31(30.4)$ & $32(31.4)$ \\
\hline si, ai & $5(4.9)$ & $1(1.0)$ \\
\hline \multicolumn{3}{|l|}{ Metastasis to lymph nodes ${ }^{\mathrm{b}}$} \\
\hline $\mathrm{n} 1(+)$ & $77(75.5)$ & $78(76.5)$ \\
\hline $\mathrm{n} 2(+)$ & $20(19.6)$ & $22(21.6)$ \\
\hline $\mathrm{n} 3(+)$ & $5(4.9)$ & $2(2.0)$ \\
\hline \multicolumn{3}{|l|}{ Histological stage } \\
\hline IIIa & $77(75.5)$ & $78(76.5)$ \\
\hline $\mathrm{IIIb}$ & $25(24.5)$ & $24(23.5)$ \\
\hline \multicolumn{3}{|l|}{ Lymphatic invasion } \\
\hline ly0 & $11(10.8)$ & $9(8.8)$ \\
\hline $\operatorname{ly}(+)$ & $91(89.2)$ & $93(91.2)$ \\
\hline \multicolumn{3}{|l|}{ Venous invasion } \\
\hline v0 & $18(17.6)$ & $20(19.6)$ \\
\hline $\mathrm{v}(+)$ & $84(82.4)$ & $82(80.4)$ \\
\hline \multicolumn{3}{|l|}{ Histological curability } \\
\hline R0 & $102(100.0)$ & $102(100.0)$ \\
\hline
\end{tabular}

${ }^{\text {a}}$ For depth of invasion, cases with $\mathrm{sm} / \mathrm{mp} / \mathrm{ss}$, a1 are categorized as T1-3. Cases with se, a2/si, ai are categorized as T4. ${ }^{\mathrm{b}}$ Metastasis to 1-3 lymph nodes is categorized as $\mathrm{n} 1$. Cases with more than 4 affected lymph nodes are categorized as $\mathrm{n} 2$. $\mathrm{sm}$, submucosa; mp, muscularis propria; ss, subserosal; a1, sub-adventitia; se, serosa; a2, adventitia; si, adjacent structures.

guish between patients with good and poor outcomes. The effects of intratumoral TP/DPD enzyme ratio on disease-free survival by tumor site, $\mathrm{T}$ and $\mathrm{N}$ category were analyzed.

The sample size was estimated assuming an HR of 2.2 for the doxifluridine group based on Nishimura et al (9), who demonstrated that disease-free survival was significantly longer in the patient cohort with a TP/DPD ratio $\geq 2.0$ with doxifluridine adjuvant therapy. An estimated 240 patients in total were required, with 120 patients in the doxifluridine group. Assuming a 5-year disease-free survival rate of $70 \%$ for patients with stage III (R0) colon cancer and 60\% for rectal cancer, a colon:rectal cancer ratio of 55:45 and a 1-year enrollment period with a 5-year follow-up period, an $\alpha$ error of 0.05 (one-sided) and detection power (1- $\beta$ ) of $63 \%$ were ensured. Data were collected by EPS Co., Ltd. Statistical analyses were conducted under the supervision of T.T., at the Center for Medical Statistics.

\section{Results}

Two hundred and four patients were enrolled at 13 institutions from January 2002 to September 2003 and 102 patients were assigned to the doxifluridine group and 102 patients to the UFT group. One hundred and two patients in the doxifluridine group and 99 patients in the UFT group received treatment as allocated, respectively. The analysis set comprised 195 patients in which TP and DPD protein levels were measured (doxifluridine, $\mathrm{n}=98$; UFT, $\mathrm{n}=97$ ). There were no differences between the 2 groups in the distribution of patient baseline characteristics (Table I).

Compliance and safety. Seventy-seven patients completed treatment and 25 patients discontinued intervention in the doxifluridine group (death, $\mathrm{n}=1$; relapsed/metastasis, $\mathrm{n}=7$; adverse events related to study drugs, $\mathrm{n}=10$; declined treatment, $\mathrm{n}=6$; other, $\mathrm{n}=1$ ). Sixty-seven patients completed treatment and 32 patients discontinued intervention in the UFT group (relapsed/metastasis, $\mathrm{n}=15$; complications, $\mathrm{n}=1$; adverse events related to study drugs, $n=13$; declined treatment, $n=2$; other, $\mathrm{n}=1$ ).

Treatment compliance was similar in both groups $(\geq 70 \%$ of treatment days completed for doxifluridine, $72.5 \%$; UFT, $68.7 \%$; $\geq 70 \%$ doses received for doxifluridine, $68.6 \%$; UFT, $65.7 \%)$.

Adverse events were reported in 61 patients $(59.8 \%)$ in the doxifluridine group and 71 patients $(71.7 \%)$ in the UFT group and grade 3 events were reported in 7 patients in both groups (one patient had grade 4 anorexia and stomach pains caused by adhesive bowel obstruction) (Table II).

Adverse events assessed as grade $\geq 2$ that resulted in treatment discontinuation or dose reduction were reported in 56 patients in the UFT group and 55 patients in the doxifluridine group (note: patients may have experienced $\geq 1$ event).

Intratumoral markers. TP and DPD protein levels were measured in 195 patients and mRNA in 170 patients. Median TP and DPD protein levels and TP, DPD, TS and OPRT mRNA levels showed no significant differences between the 2 groups (Table III).

Assessment of marker predictiveness. For the effect of intratumoral TP/DPD enzyme ratio (cut-off value 2.0) on disease-free survival in the doxifluridine group, the primary study endpoint, there was no statistically significant difference between the 2 cohorts in the 5-year disease-free survival rate: $\mathrm{TP} / \mathrm{DPD}$ ratio $\geq 2.0,71.4 \%$ (95\% CI 59.3-80.5) vs. TP/DPD ratio $<2.0,66.5 \%(95 \%$ CI $45.4-80.9)(\log$-rank $\mathrm{P}=0.6850)$ (Fig. 1). 
Table II. Adverse events graded according to the Common Toxicity Criteria of the National Cancer Institute (version 2).

\begin{tabular}{|c|c|c|c|c|c|c|c|c|c|c|}
\hline & \multicolumn{5}{|c|}{ Doxifluridine $(\mathrm{n}=102)$} & \multicolumn{5}{|c|}{ Uracil/tegafur $(\mathrm{n}=99)$} \\
\hline & G1 & G2 & G3 & G4 & Total & G1 & G2 & G3 & G4 & Total \\
\hline Nausea & 3 & 4 & & & 7 & 4 & 4 & 2 & & 10 \\
\hline Vomiting & & & & & & & 2 & 1 & & 3 \\
\hline Anorexia & 2 & 5 & 1 & & 8 & 13 & 5 & 1 & $1^{\mathrm{b}}$ & 20 \\
\hline Diarrhea & 7 & 4 & 2 & & 13 & 4 & 5 & 1 & & 10 \\
\hline Stomatitis & 5 & 1 & & & 6 & 2 & & & & 2 \\
\hline Alopecia & 1 & & & & 1 & 2 & & & & 2 \\
\hline Pigmentation change & 3 & & & & 3 & 4 & & & & 4 \\
\hline Rash/desquamation & 1 & 4 & & & 5 & 4 & 7 & & & 11 \\
\hline Fatigue $^{\mathrm{a}}$ & 5 & 2 & 1 & & 8 & 8 & 4 & 1 & & 13 \\
\hline Hematuria & 3 & & & & 3 & 2 & & & & 2 \\
\hline Increased creatinine & 3 & & & & 3 & 2 & & & & 2 \\
\hline Decreased WBC & 7 & 3 & & & 10 & 4 & 7 & & & 11 \\
\hline Decreased platelet count & 9 & & & & 9 & 4 & & & & 4 \\
\hline Decreased hemoglobin & 7 & 6 & 1 & & 14 & 13 & 7 & & & 20 \\
\hline Increased bilirubin & 15 & 13 & & & 28 & 22 & 3 & & & 25 \\
\hline Increased GOT/GPT & 11 & 3 & 1 & & 15 & 22 & 5 & 2 & & 29 \\
\hline Other & 4 & 2 & 2 & & $10^{\mathrm{c}}$ & 6 & 1 & 1 & $1^{\mathrm{b}}$ & $11^{\mathrm{d}}$ \\
\hline
\end{tabular}

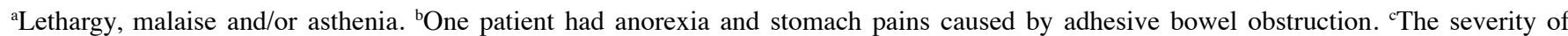
2 of 10 patients not determined by grade. ${ }^{\mathrm{d}}$ The severity of 2 of 11 patients not determined by grade. GPT, glutamic-pyruvic transaminase; GOT, glutamic-oxaloacetic transaminase; WBC, white blood cell count.

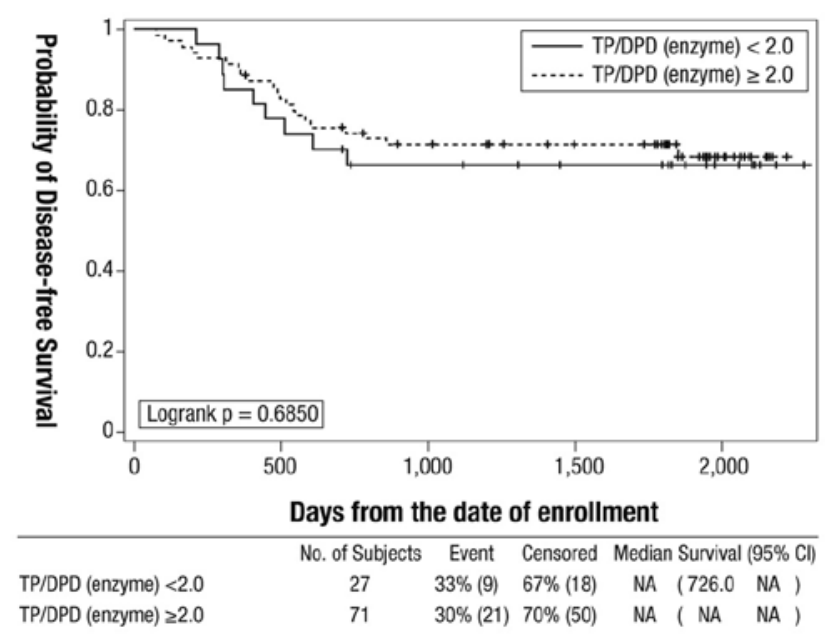

Figure 1. Disease-free survival by thymidine phosphorylase/dihydropyrimidine dehydrogenase (TP/DPD) ratio (cut-off value 2.0) in patients treated with doxifluridine $(\mathrm{n}=98)$.

Results for the secondary endpoints were as follows: i) The effect of the magnitude of intratumoral TP/DPD enzyme ratio on disease-free survival with oral fluoropyrimidine treatment was only significant $(\mathrm{HR}=2.76, \mathrm{P}=0.00469)$ when analyzed in the model including the drug and the parameter and the interactions between them. Proportional hazards analysis showed that the effects of TP and DPD protein levels on disease-free survival and the effects of the parameters (i.e. TP, DPD protein levels and TP/DPD enzyme ratio) on overall survival were not significant in the models (data not shown).

The 5-year disease-free survival rate was statistically significantly higher in the cohort with a higher intratumoral TP/DPD enzyme ratio (median $\geq 2.63$ ) compared to the cohort with a lower TP/DPD ratio (<2.63): $71.9 \%$ (95\% CI 61.4-80.0) vs. 57.0\% (95\% CI 46.3-66.3) (log-rank $\mathrm{P}=0.0277$ ) (Fig. 2A). The effects of the magnitude of the TP/DPD ratio on diseasefree survival in each of the doxifluridine and UFT groups are shown in Fig. 2. In the UFT group, the 5-year diseasefree survival rate was statistically significantly higher in the cohort with the higher TP/DPD ratio (median $\geq 2.63$ ) than in the cohort with a lower TP/DPD ratio (median $<2.63$ ): $74.4 \%$ (95\% CI 58.2-85.1) vs. 44.1\% (95\% CI 29.7-57.6) (log-rank $\mathrm{P}=0.0029$ ) (Fig. 2C). In the doxifluridine group, the effect of the magnitude of the TP/DPD ratio on disease-free survival was not statistically significant (log-rank $\mathrm{P}=0.9541$ ) (Fig. 2B).

ii) Proportional hazard analysis demonstrated that the effects of the parameters (i.e. intratumoral TP, DPD, TS and OPRT mRNA levels and TP/DPD mRNA ratio) on diseasefree and overall survival were not significant in any of the models (data not shown).

iii) Proportional hazards analysis showed that the effects of the parameters (i.e. TS tandem repeat type $2 \mathrm{R} / 2 \mathrm{R}, 2 \mathrm{R} / 3 \mathrm{R}$ or $3 R / 3 R$ ) on disease-free and overall survival were not significant in the models (data not shown).

Exploratory analyses. An exploratory Cox regression analysis identified a cut-off value of 3.1 for the intratumoral TP/DPD 
Table III. Intratumoral markers.

\begin{tabular}{|c|c|c|}
\hline & \multicolumn{2}{|c|}{ Median (range) } \\
\hline & Doxifluridine $(\mathrm{n}=98)$ & Uracil/tegafur $(n=97)$ \\
\hline \multicolumn{3}{|c|}{ Protein levels (U/mg protein) } \\
\hline $\mathrm{TP}$ & $81.3(13.2-242.5)$ & $80.3(13.7-301.3)$ \\
\hline DPD & $34.4(4.9-124.2)$ & $32.8(8.8-139.8)$ \\
\hline \multirow[t]{2}{*}{ TP/DPD } & $2.663(0.54-6.94)$ & $2.569(0.75-9.57)$ \\
\hline & Doxifluridine $(n=85)$ & Uracil/tegafur $(n=85)$ \\
\hline \multicolumn{3}{|c|}{ mRNA levels (copy number) ${ }^{\mathrm{a}}$} \\
\hline $\mathrm{TP}$ & $2.61(0.21-21.49)$ & $2.93(0.24-141.16)$ \\
\hline DPD & $0.13(0.02-1.92)$ & $0.18(0.006-1.57)$ \\
\hline TP/DPD & $19.47(2.19-145.66)$ & $22.06(1.32-558.16)$ \\
\hline TS & $1.06(0.23-14.86)$ & $1.17(0.04-20.40)$ \\
\hline OPRT & $0.82(0.04-7.37)$ & $0.88(0.182-6.18)$ \\
\hline
\end{tabular}

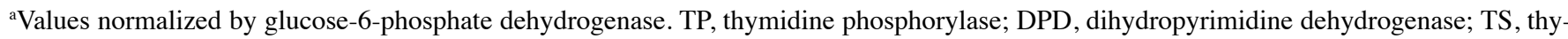
midylate synthase; OPRT, orotate phosphoribosyltransferase.

enzyme ratio to distinguish between patients with good and poor outcomes with oral fluoropyrimidine treatment. The 5 -year disease-free survival rates in cohorts with TP/DPD ratios $\geq 3.1$ and $<3.1$ were 77.7 and $56.1 \%$, respectively (logrank $\mathrm{P}=0.0059$ ) (Fig. 3). Multiple regression analysis using Cox's method identified T4 $(\mathrm{P}=0.0068 ; \mathrm{HR}=2.225 ; 95 \% \mathrm{CI}$ 1.247-3.969) and intratumoral TP/DPD enzyme ratio $\geq 3.1$ $(\mathrm{P}=0.0035 ; \mathrm{HR}=0.394 ; 95 \% \mathrm{CI} 0.211-0.735)$ as independent variables for prognosis. The P-values for other variables examined in the model (i.e. $\mathrm{N}$ category, tumor site, drug, TP/ DPD mRNA ratio) were not statistically significant (data not shown).

Additionally, exploratory analyses to examine the effect of the intratumoral TP/DPD enzyme ratio on disease-free survival by tumor site, $\mathrm{T}$ stage and $\mathrm{N}$ stage in patients treated with oral fluoropyrimidines were conducted (Fig. 4). Analysis by tumor site displayed that the TP/DPD ratio was significantly associated with the disease-free survival (log-rank $\mathrm{P}=0.0033$ ) in patients with colon cancer. Analysis by $\mathrm{T}$ category displayed an association between TP/DPD ratio and disease-free survival ( $\log$-rank $\mathrm{P}=0.0039)$ in patients with T4, but not T1-3. Analysis by $\mathrm{N}$ category displayed an association between TP/DPD ratio and disease-free survival (log-rank $\mathrm{P}=0.0224)$ in patients with $\mathrm{N} 1$, but without $\mathrm{N} 2$ disease.

\section{Discussion}

In this study, we examined several biomarkers for their possible predictive value in relation to the outcome following adjuvant chemotherapy with oral fluoropyrimidines in patients with stage III colorectal cancer in order to identify subgroups of patients who may benefit from this intervention. Our findings on the predictive value of biomarkers in tumor cells and blood samples were unexpected.
The TP/DPD ratio is significantly higher in cancer cell lines with high sensitivity for doxifluridine and capecitabine compared to those with lower sensitivity (8). In addition, synergistic effects were observed when doxifluridine was used in combination with chemotherapeutic agents or radiotherapy in vivo, since tumor TP levels are increased by these treatments (15). This synergistic antitumor activity, which results from TP upregulation, is specific for doxifluridine, not for UFT or 5-FU (15). These data suggest that TP may be a potential factor for determining the outcome following doxifluridine treatment. There was a significant association between the magnitude of intratumoral TP/DPD enzyme ratio and disease-free survival $(\mathrm{HR}=2.76 ; \mathrm{P}=0.00469)$ when the interaction between the drug and this parameter was analyzed. The 5-year disease-free survival rate in the group with a high TP/DPD ratio (median $\geq 2.63$ ) was statistically significantly higher compared to the group with a low TP/DPD ratio (median $<2.63$; log-rank $\mathrm{P}=0.0277$ ).

However, the effect of the intratumoral TP/DPD enzyme ratio (cut-off value both 2.0 and median of 2.63) on disease-free survival was not statistically significant in the doxifluridine group (log-rank $\mathrm{P}=0.6850,0.9541$, respectively). TP levels measured using tumor samples were found to be more important for doxifluridine (and capecitabine) compared to using normal or blood samples, since only fluoropyrimidine converted by TP in the tumor has a direct effect $(16,17)$. For measurement, tissues which included both the tumor and stroma cells were used, since fluoropyrimidine converted in cells with high TP expression near the tumor may also have a tumor response (18). Unexpected results may be caused by TP levels not being stable before and after treatment initiation, since TP is induced by a number of cytokines $(19,20)$. TP levels were measured from resected primary lesion tumors and outcomes were analyzed 5 years after treatment initiation, 
A

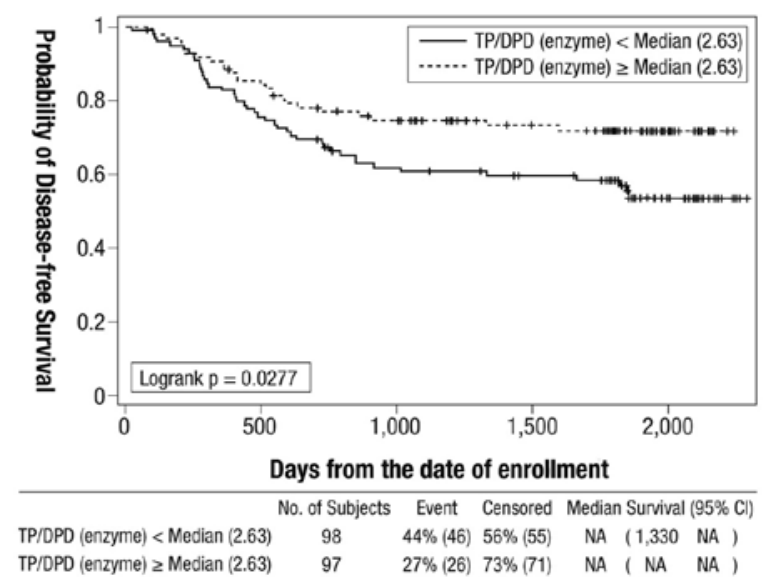

B

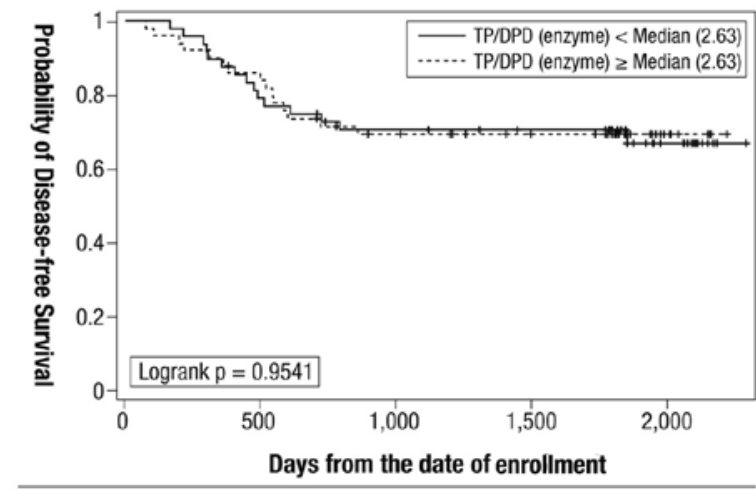

\begin{tabular}{ccccc}
\hline No. of Subjects Event & Censored Median Survival (95\% Cl) \\
TP/DPD (enzyme) < Median (2.63) & 48 & $31 \%(15)$ & $69 \%(33)$ & NA ( NA NA )
\end{tabular} TP/DPD (enzyme) z Median (2.63) $50 \quad 30 \%(15) 70 \%(35) \quad$ NA ( NA NA )

C

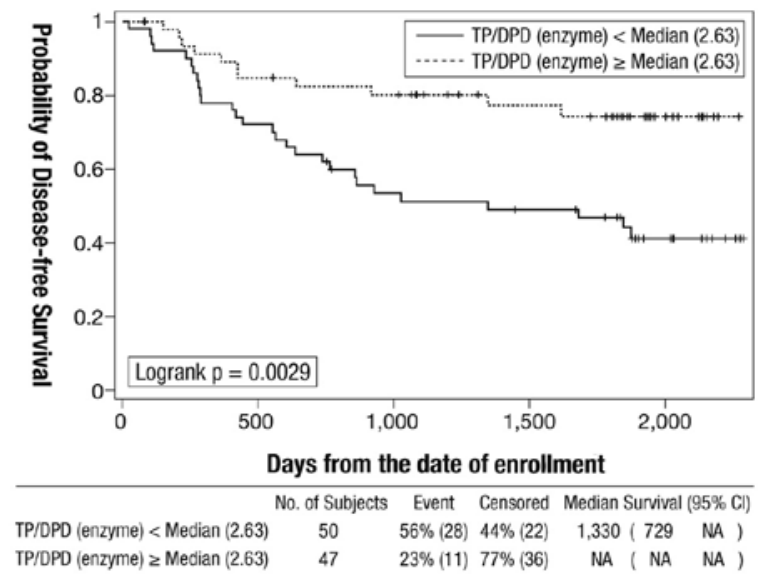

Figure 2. Disease-free survival according to thymidine phosphorylase/ dihydropyrimidine dehydrogenase (TP/DPD) ratio (using median value as cut-off, i.e. 2.63) in patients treated with (A) oral fluoropyrimidines $(n=195)$, (B) doxifluridine $(\mathrm{n}=98)$ and (C) UFT $(\mathrm{n}=97)$.

which may explain the different outcome from the in vivo study (8).

The effect of the intratumoral TP/DPD enzyme ratio (cut-off median of 2.63) on disease-free survival was statistically significant in the UFT group (log-rank $\mathrm{P}=0.0029)$. UFT confers its effect by maintaining high fluoropyrimidine levels in the blood and not in the tumor. However, since TP levels in

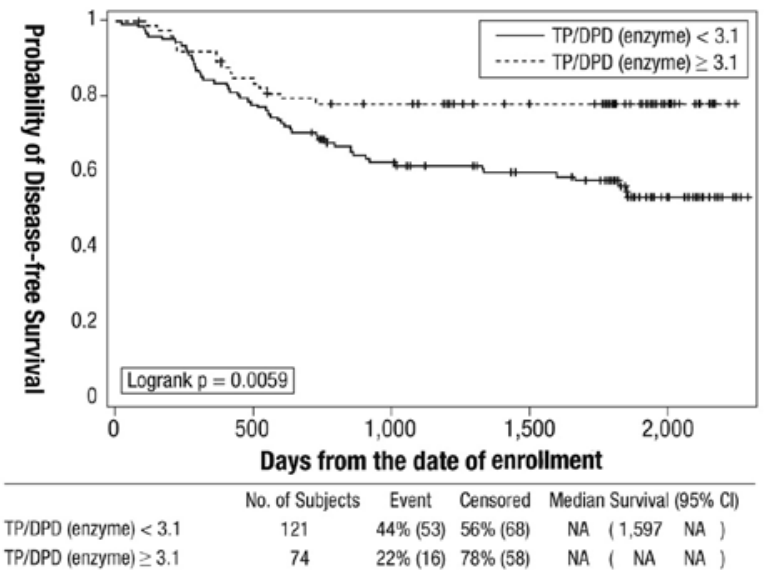

Figure 3. Disease-free survival according to the thymidine phosphorylase/ dihydropyrimidine dehydrogenase (TP/DPD) ratio (using cut-off value identified by Cox regression analysis, 3.1) in patients treated with oral fluoropyrimidines $(\mathrm{n}=195)$.

the tumor catalyze the conversion between fluoropyrimidine and 2'-deoxy-5-fluorouridine (FUdR) [precursor of 5-fluoro2'-deoxyuridine-5'-monophosphate (FdUMP) which has an antitumor effect] and high TP levels produce deoxy ribose1-phosphate $(\mathrm{dR} 1 \mathrm{P})$ from thymidine (dR1P promotes the conversion between fluoropyrimidine and FUdR) (21), high TP expression may be meaningful for UFT.

The association between TS, OPRT and the sensitivity to fluoropyrimidine drugs has been observed in previous research $(10,22-25)$. It has also been reported that combined analysis, such as analysis with TS and TP, DPD, with OPRT and DPD may predict efficacy or outcome of treatment with fluoropyrimidines more precisely $(10,26-28)$. In the present study we did not demonstrate an association between any factors other than the TP/DPD enzyme ratio and the efficacy of fluoropyrimidine drugs.

For TS tandem repeat type, it was previously observed that the level of expression of the TS protein is higher and that fluoropyrimidine drugs are less efficacious in patients with the $3 R / 3 R$ allele compared to those with $2 R / 2 R$ or $2 R / 3 R$ alleles (29). Previous research reported that TS tandem polymorphisms are potentially predictive biomarkers, not only of response, but also for the occurrence of adverse events. Conversely, other research has claimed that TS-tandem polymorphisms are not associated with the efficacy of fluoropyrimidine drugs (30). The results of our study were negative for the possibility that TS-tandem type is potentially predictive of an outcome following oral fluoropyrimidine adjuvant therapy.

Our study has several important design features. First, since this study targeted stage III cancer, the number of residual cancer cells persisting after standard curative resection may have had a major effect on postoperative survival. For this reason, the skill of the surgeon is a major factor. Since the institutions participating in our study belonged to the Japanese Society for Cancer of the Colon and Rectum, we hypothesize that surgeon-related factors were minimized. Other factors which may have influenced our findings were potential differences in the timing of the collection and handling of tissue specimens and the differences between individuals in medication compliance. Participating sites were rigorously drilled in the methodology for the collection of specimens. Institutions 
A

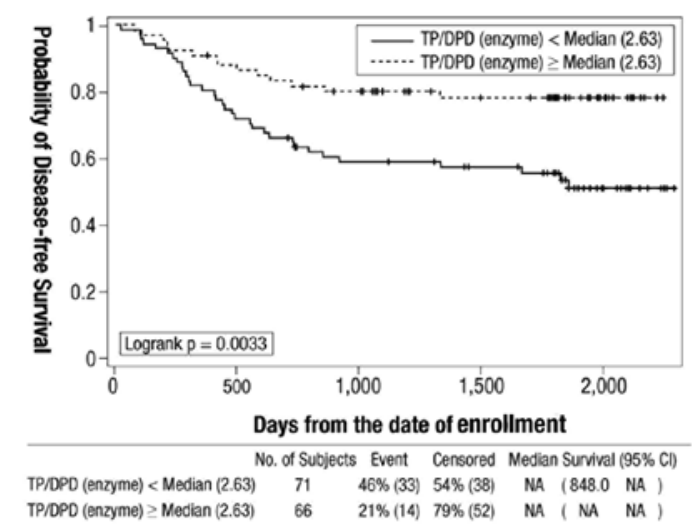

C

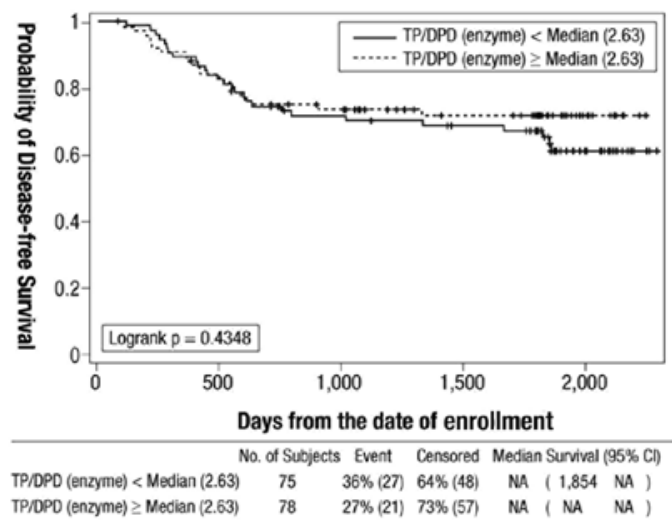

E

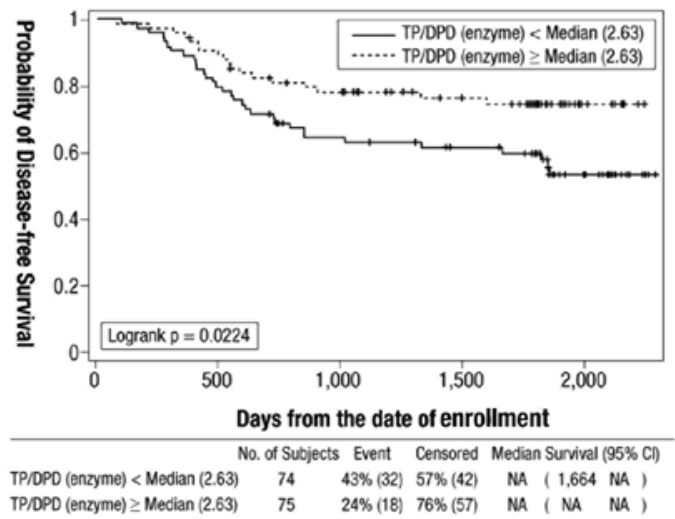

B

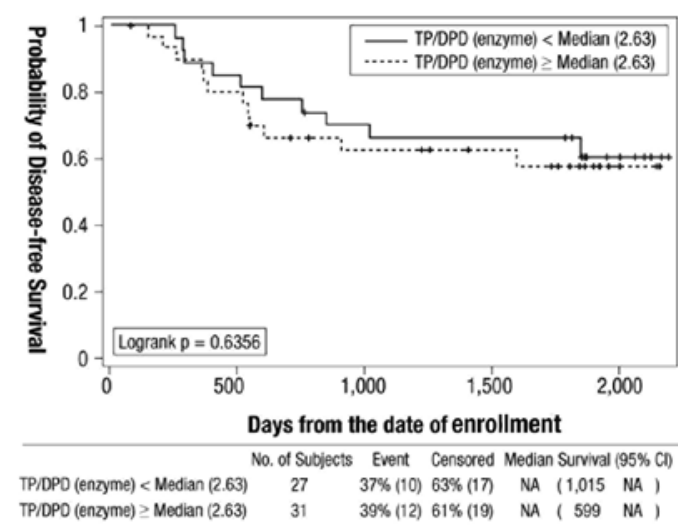

D

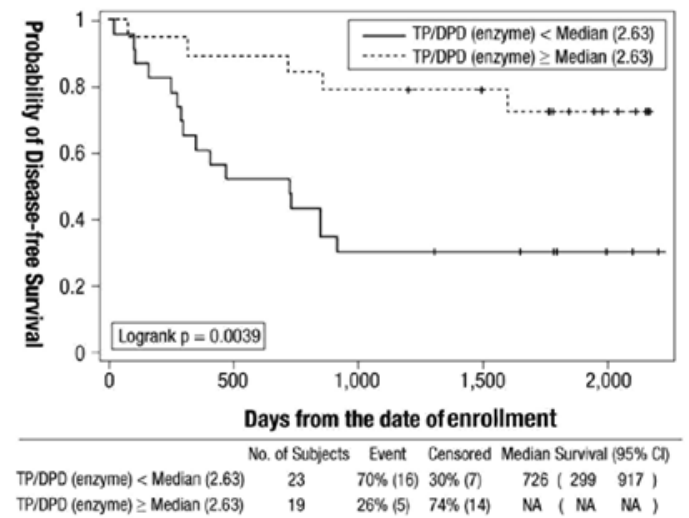

$\mathrm{F}$

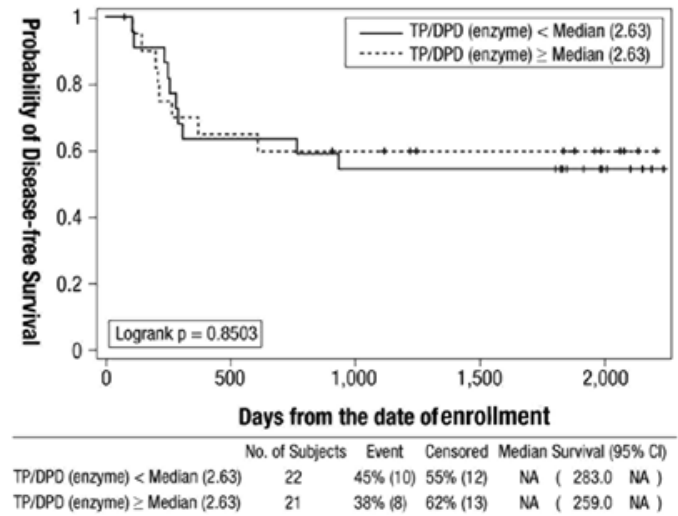

Figure 4. Disease-free survival according to the thymidine phosphorylase/dihydropyrimidine dehydrogenase (TP/DPD) ratio (using median value as cut-off, i.e. 2.63) by tumor type $[(A)$ colon cancer; (B) rectal cancer], T stage [(C) T1-3; (D) T4] and N stage $[(E) \mathrm{N} 1 ;(\mathrm{F}) \mathrm{N} 2]$ in patients treated with oral fluoropyrimidines.

which returned specimens with considerable degradation during the study were provided with on-site guidance and the requirement for prompt processing was reinforced. Medication compliance was assessed every quarter by the investigator by directly interviewing each patient. Patients were asked for detailed reasons in the event of interruption or discontinuation of the medication. We hypothesize that biases in this study were minimal as a result of these measures.

Our study has some limitations. One of the most serious issues is that there was no control arm (i.e. surgery-alone group). TP is identical to platelet-derived endothelial cell growth factor (PD-ECGF) (31) and patients with high levels of tumoral TP expression have a poor prognosis $(32,33)$. This may be important, since patients in the high-TP group may be especially responsive to doxifluridine (9), although there were no significant differences compared with UFT in our study. This may indicate that TP is a prognostic, as well as a predictive, marker. A surgery-alone group may have helped to clarify this issue and explain our unexpected results. In conclusion, the magnitude of the intratumoral TP/DPD enzyme ratio may predict outcomes in patients with stage III colorectal cancer who are treated with adjuvant chemotherapy with oral fluoropyrimidines. The intratumoral TP/DPD enzyme ratio may therefore allow the individualization of postoperative oral fluoropyrimidine adjuvant therapy in stage III colorectal cancer. Further exploration and verification of the magnitude 
of the intratumoral TP/DPD enzyme ratio as a biomarker is required.

\section{Acknowledgements}

The authors thank all the investigators who participated in this trial: Kazuo Hase, National Defense Medical College Hospital, Tokorozawa; Naohiro Tomita, Kansai Rosai Hospital (now transferred to Hyogo College of Medicine), Amagasaki; Makoto Akaike, Kanagawa Cancer Center, Yokohama; Akihiko Murata, Hirosaki University School of Medicine and Hospital, Hirosaki; Kazuo Shirouz, Kurume University School of Medicine, Kurume and Yozi Nishimura, Saitama Cancer Center, Ina-cho, Saitama, Japan. We also wish to thank Chugai Pharmaceutical Co., Ltd Research Center, Kamakura, for providing tumor tissue assay and analysis and EPS Co., Ltd, Tokyo, for clinical data collection. Funding support was provided by the Japan-China Medical Association.

\section{References}

1. Center for Cancer Control and Information Services, National Cancer Center, Japan: http://ganjoho.ncc.go.jp/public/statistics/ index.html. Accessed August 16, 2011.

2. Efficacy of adjuvant fluorouracil and folinic acid in colon cancer International Multicentre Pooled Analysis of Colon Cancer Trials (IMPACT) investigators. Lancet 345: 939-944, 1995.

3. Haller DG, Catalano PJ, Macdonald JS, et al: Phase III study of fluorouracil, leucovorin, and levamisole in high-risk stage II and III colon cancer: final report of Intergroup 0089. J Clin Oncol 23: 8671-8678, 2005.

4. Comparison of fluorouracil with additional levamisole, higher-dose folinic acid, or both, as adjuvant chemotherapy for colorectal cancer: a randomised trial. QUASAR Collaborative Group. Lancet 355: 1588-1596, 2000.

5. André T, Quinaux E, Louvet C, et al: Phase III study comparing a semimonthly with a monthly regimen of fluorouracil and leucovorin as adjuvant treatment for stage II and III colon cancer patients: final results of GERCOR C96.1. J Clin Oncol 25: 3732-3738, 2007.

6. Twelves C, Wong A, Nowacki MP, et al: Capecitabine as adjuvant treatment for stage III colon cancer. N Engl J Med 352: 2696-2704, 2005.

7. Lembersky BC, Wieand HS, Petrelli NJ, et al: Oral uracil and tegafur plus leucovorin compared with intravenous fluorouracil and leucovorin in stage II and III carcinoma of the colon: results from National Surgical Adjuvant Breast and Bowel Project Protocol C-06. J Clin Oncol 24: 2059-2064, 2006.

8. Ishikawa T, Sekiguchi F, Fukase Y, et al: Positive correlation between the efficacy of capecitabine and doxyfluridine and the ratio of thymidine phosphorylase to dihydropyrimidine dehydrogenase activities in tumors in human cancer xenografts. Cancer Res 58: 685-690, 1998.

9. Nishimura G, Terada I, Kobayashi T, et al: Thymidine phosphorylase and dihydropyrimidine dehydrogenase levels in primary colorectal cancer show a relationship to clinical effects of 5'-deoxy-5-fluorouridine as adjuvant chemotherapy. Oncol Rep 9: 479-482, 2002.

10. Ichikawa W, Uetake H, Shirota Y, et al: Both gene expression for orotate phosphoribosyltransferase and its ratio to dihydropyrimidine dehydrogenase influence outcome following fluoropyrimidine-based chemotherapy for metastatic colorectal cancer. Br J Cancer 89: 1486-1492, 2003.

11. Sakamoto J, Ohashi Y, Hamada C, et al: Efficacy of oral adjuvant therapy after resection of colorectal cancer: 5 -year results from three randomized trials. J Clin Oncol 22: 484-492, 2004.

12. Nishida M, Hino A, Mori K, et al: Preparation of anti-human thymidine phosphorylase monoclonal antibodies useful for detecting the enzyme levels in tumor tissues. Biol Pharm Bull 19: $1407-1411,1996$.

13. Mori K, Hasegawa M, Nishida M, et al: Expression levels of thymidine phosphorylase and dihydropyrimidine dehydrogenase in various human tumor tissues. Int J Oncol 17: 33-38, 2000.
14. Kawakami K and Watanabe G: Identification and functional analysis of single nucleotide polymorphism in the tandem repeat sequence of thymidylate synthase gene. Cancer Res 63: 6004-6007, 2003.

15. Sawada N, Ishikawa T, Fukase Y, et al: Induction of thymidine phosphorylase activity and enhancement of capecitabine efficacy by taxol/taxotere in human cancer xenografts. Clin Cancer Res 4: 1013-1019, 1998.

16. Gieschke R, Burger HU, Reigner B, et al: Population pharmacokinetics and concentration-effect relationships of capecitabine metabolites in colorectal cancer patients. Br J Clin Pharmacol 55: 252-263, 2003.

17. Patterson AV, Zhang H, Moghaddam A, et al: Increased sensitivity to the prodrug 5'-deoxy-5-fluorouridine and modulation of 5-fluoro-2'-deoxyuridine sensitivity in MCF-7 cells transfected with thymidine phosphorylase. Br J Cancer 72: 669-675, 1995.

18. Evrard A, Cuq P, Ciccolini J, et al: Increased cytotoxicity and bystander effect of 5-fluorouracil and 5-deoxy-5-fluorouridine in human colorectal cancer cells transfected with thymidine phosphorylase. Br J Cancer 80: 1726-1733, 1999.

19. Toi M, Rahman MA, Bando $\mathrm{H}$ and Chow LW: Thymidine phosphorylase (platelet-derived endothelial-cell growth factor) in cancer biology and treatment. Lancet Oncol 6: 158-166, 2005.

20. Eda H, Fujimoto K, Watanabe S, et al: Cytokines induce thymidine phosphorylase expression in tumor cells and make them more susceptible to 5'-deoxy-5-fluorouridine. Cancer Chemother Pharmacol 32: 333-338, 1993.

21. Miyadera K, Sumizawa T, Haraguchi M, et al: Role of thymidine phosphorylase activity in the angiogenic effect of platelet-derived endothelial cell growth factor/thymidine phosphorylase. Cancer Res 55: 1687-1690, 1995.

22. Lenz HJ, Hayashi K, Salonga D, et al: p53 point mutations and thymidylate synthase messenger RNA levels in disseminated colorectal cancer: an analysis of response and survival. Clin Cancer Res 4: 1243-1250, 1998.

23. Paradiso A, Simone G, Petroni S, et al: Thymidilate synthase and p53 primary tumour expression as predictive factors for advanced colorectal cancer patients. Br J Cancer 82: 560-567, 2000.

24. Sinicrope FA, Rego RL, Halling KC, et al: Thymidylate synthase expression in colon carcinomas with microsatellite instability. Clin Cancer Res 12: 2738-2744, 2006.

25. Ochiai T, Nishimura K, Noguchi H, et al: Prognostic impact of orotate phosphoribosyl transferase among 5-fluorouracil metabolic enzymes in resectable colorectal cancers treated by oral 5-fluorouracil-based adjuvant chemotherapy. Int J Cancer 118: 3084-3088, 2006.

26. Salonga D, Danenberg KD, Johnson M, et al: Colorectal tumors responding to 5-fluorouracil have low gene expression levels of dihydropyrimidine dehydrogenase, thymidylate synthase, and thymidine phosphorylase. Clin Cancer Res 6: 1322-1327, 2000.

27. Lassmann S, Hennig M, Rosenberg R, et al: Thymidine phosphorylase, dihydropyrimidine dehydrogenase and thymidylate synthase mRNA expression in primary colorectal tumors correlation to tumor histopathology and clinical follow-up. Int J Colorectal Dis 21: 238-247, 2006.

28. Ciaparrone M, Quirino M, Schinzari G, et al: Predictive role of thymidylate synthase, dihydropyrimidine dehydrogenase and thymidine phosphorylase expression in colorectal cancer patients receiving adjuvant 5-fluorouracil. Oncology 70: 366-377, 2006.

29. Park DJ, Stoehlmacher J, Zhang W, et al: Thymidylate synthase gene polymorphism predicts response to capecitabine in advanced colorectal cancer. Int J Colorectal Dis 17: 46-49, 2002.

30. Park CM,Lee WY, Chun HK, et al: Relationship of polymorphism of the tandem repeat sequence in the thymidylate synthase gene and the survival of stage III colorectal cancer patients receiving adjuvant 5-flurouracil-based chemotherapy. J Surg Oncol 101: 22-27, 2010.

31. Goto $\mathrm{H}$, Kohno K, Sone $\mathrm{S}$, et al: Interferon gamma-dependent induction of thymidine phosphorylase/platelet-derived endothelial growth factor through gamma-activated sequence-like element in human macrophages. Cancer Res 61: 469-473, 2001.

32. Takebayashi Y, Akiyama S, Akiba S, et al: Clinicopathologic and prognostic significance of an angiogenic factor, thymidine phosphorylase, in human colorectal carcinoma. J Natl Cancer Inst 88: 1110-1117, 1996.

33. Tokunaga Y, Hosogi H, Hoppou T, et al: Prognostic value of thymidine phosphorylase/platelet-derived endothelial cell growth factor in advanced colorectal cancer after surgery: evaluation with a new monoclonal antibody. Surgery 131: 541-547, 2002. 